\title{
Survey of fruit nutrient removal by mango (Mangifera indica L.) cultivars for the export market in various producing regions of Mexico Evaluación de la remoción de nutrimentos por el fruto de cultivares de mango (Mangifera indica L.) para el mercado de exportación en varias regiones productoras de México
}

\author{
Adriana Mellado-Vázquez ${ }^{1 \oplus}$, Samuel Salazar-García $a^{1 \notin}{ }^{\circledR}$, \\ Ricardo Goenaga ${ }^{2}$, and Alfredo López-Jiménez ${ }^{3}{ }^{\circledR}$
}

\begin{abstract}
${ }^{1}$ INIFAP, Campo Experimental Santiago Ixcuintla. Entronque carretera Internacional México-Nogales km 6. 63300 Santiago Ixcuintla, Nayarit, México. * Corresponding autor (salazar.avocado@gmail.com)

${ }^{2}$ USDA-ARS, Tropical Agriculture Research Station. 2200 P. A. Campos Ave., Suite 201. 00680-5470 Mayagüez, Puerto Rico, USA.

${ }^{3}$ Postgrado en Recursos Genéticos y Productividad-Fruticultura, Colegio de Postgraduados, Campus Montecillo. Carretera México-Texcoco km 36.5, Montecillo. 56230 Texcoco, Estado de México, México.
\end{abstract}

\section{SUMMARY}

In Mexico there are more than 201400 ha grown with different mango (Mangifera indica L.) cultivars. This may cause variations in mineral requirement, fruit mineral concentrations and nutrient removal. The objective of this research was to make a survey of mineral concentration in fruit tissues and calculate nutrient removal by fruit tissues during harvest of the most important mango cultivars (Ataulfo, Kent and Tommy Atkins) from several production regions (Campeche, Chiapas, Oaxaca, Nayarit, and Sinaloa) of Mexico. Fruit at physiological maturity were harvested from commercial mango orchards and concentration of nitrogen $(\mathrm{N})$, phosphorus $(\mathrm{P})$, potassium $(\mathrm{K})$, calcium $(\mathrm{Ca})$, magnesium $(\mathrm{Mg})$, sulfur $(\mathrm{S})$, iron $(\mathrm{Fe})$, copper $(\mathrm{Cu})$, manganese $(\mathrm{Mn})$, zinc $(\mathrm{Zn})$ and boron $(\mathrm{B})$ was determined for skin, mesocarp, endocarp, and seed tissues. Each tissue was cut into thin slices and they were dehydrated in a forced air oven at $70{ }^{\circ} \mathrm{C}$, after that, were pulverized and they were analyzed: nitrogen by semi-microKjeldahl digestion, phosphorus with the ascorbic acid method and the other nutrients with atomic absorption. The removal of nutrients was calculated considering the weight of the fruit and the content of nutrients in each tissue. Significant differences in the concentration of $\mathrm{N}, \mathrm{K}, \mathrm{Mg}$, and $\mathrm{Zn}$ were found among cultivars and tissues. Concentration

Recommended citation:

Mellado-Vázquez, A., S. Salazar-García, R. Goenaga, and A. López-Jiménez. 2019. Survey of fruit nutrient removal by mango (Mangifera indica L.) cultivars for the export market in various producing regions of Mexico. Terra Latinoamericana 37: 437-447.

DOI: https://doi.org/10.28940/terra.v37i4.528 of $\mathrm{P}, \mathrm{S}, \mathrm{Cu}$, and $\mathrm{Mn}$ in the skin, $\mathrm{Ca}, \mathrm{Cu}$, and $\mathrm{Mn}$ in the mesocarp, $\mathrm{Ca}, \mathrm{S}, \mathrm{Mn}$, and $\mathrm{B}$ in endocarp, and $\mathrm{S}, \mathrm{Fe}$, and $\mathrm{Mn}$ in the seed were not affected by mango cultivar. Production region affected concentration of minerals in 'Ataulfo' fruit more than in 'Tommy Atkins' and 'Kent'. Nutrient removal by mango fruit tissues was little affected in cvs. Ataulfo, Tommy Atkins and Kent. The regions with the greatest nutrient removal were Oaxaca, Campeche and Sinaloa for 'Ataulfo', 'Tommy Atkins' and 'Kent', respectively.

Index words: endocarp, mesocarp, nutrient concentration, seed, skin.

\section{RESUMEN}

En México hay más de 201400 ha plantadas con diferentes cultivares de mango (Mangifera indica L.). Los requerimientos nutrimentales, la concentración de nutrientes en el fruto y la remoción de nutrimentos por la cosecha puede ser diferente para cada cultivar. E1 objetivo de esta investigación fue realizar un estudio de la concentración de nutrimentos en los tejidos del fruto y calcular la remoción de nutrimentos por la cosecha, para los cultivares de mango más importantes (Ataulfo, Kent y Tommy Atkins), en diferentes regiones de producción (Campeche, Chiapas, Oaxaca, Nayarit, y Sinaloa) de México. Se cosecharon frutos en madurez 
fisiológica de huertos comerciales y se determinó la concentración de nitrógeno $(\mathrm{N})$, fósforo $(\mathrm{P})$, potasio $(\mathrm{K})$, calcio $(\mathrm{Ca})$, magnesio $(\mathrm{Mg})$, azufre $(\mathrm{S})$, hierro $(\mathrm{Fe})$, cobre $(\mathrm{Cu})$, manganeso $(\mathrm{Mn})$, zinc $(\mathrm{Zn})$ y boro $(\mathrm{B})$ en la piel, mesocarpio, endocarpio y semilla. Cada tejido se cortó en rebanadas delgadas que fueron deshidratadas en un horno con aire forzado a $70^{\circ} \mathrm{C}$, posteriormente se pulverizaron y se analizó nitrógeno mediante digestión semi-microKjeldahl, fósforo con el método del ácido ascórbico y los demás nutrimentos por absorción atómica. La remoción de nutrimentos se calculó considerando el peso de los frutos y el contenido de nutrimentos en cada tejido. Se encontraron diferencias significativas en la concentración de $\mathrm{N}, \mathrm{K}, \mathrm{Mg}$ y Zn entre cultivares y tejidos. Las concentraciones de $\mathrm{P}, \mathrm{S}$, $\mathrm{Cu}$ y $\mathrm{Mn}$ en la piel, $\mathrm{Ca}, \mathrm{Cu}$ y $\mathrm{Mn}$ en el mesocarpio, $\mathrm{Ca}, \mathrm{S}, \mathrm{Mn}$ y $\mathrm{B}$ en el endocarpio, y S, Fe y Mn en la semilla no fueron afectadas por el cultivar. La región productora afectó la concentración de nutrimentos en el fruto de 'Ataulfo' más que en 'Tommy Atkins' y 'Kent'. La remoción de nutrimentos por los tejidos del fruto fue poco afectada en los cvs. Ataulfo, Tommy Atkins y Kent. Las regiones con mayor remoción de nutrimentos fueron Oaxaca, Campeche y Sinaloa para 'Ataulfo', 'Tommy Atkins' y 'Kent', respectivamente.

Palabras clave: endocarpio, mesocarpio, concentración de nutrimentos, semilla, piel.

\section{INTRODUCTION}

In Mexico, mango is grown either under irrigation (84 $207 \mathrm{ha})$ or rainfed (117 $256 \mathrm{ha}$ ) conditions. The most important cultivars are: 'Ataulfo' (62 $136 \mathrm{ha}$ ), 'Manila' (33 573 ha), 'Tommy Atkins' (24 907 ha), 'Haden' (23 356 ha), 'Kent' (23 994 ha), and 'Keitt' (10 411 ha) (SIAP, 2017). For each of these cultivars, the production area under rainfed condition is $38 \%$, $17 \%, 10 \%, 4 \%, 9 \%$, and $9 \%$, respectively.

Besides irrigation management, mango orchards in Mexico are established in a range of soil types. In the Pacific mango producing region, Vertisol, Litosol, Cambisol, Luvisol, and Regosol soils predominate, while in the Yucatan peninsula, Rendzina and Solonchak soils are typical soils (IUSS, 2007).

Leaf mineral concentrations are affected by several factors. In Nayarit, Mexico, the evolution of foliar macro and micronutrients varied among Ataulfo, Kent and Tommy Atkins cultivars, and it was influenced by leaf age, vegetative flush, and tree phenology (CastroLópez et al., 2012). In an organic mango orchard in Veracruz, Mexico, leaf nutrient concentrations were also affected by cultivar and tree phenology. Before flowering, $\mathrm{N}$ concentration was higher in 'Tommy Atkins', Ca in 'Ataulfo' and $\mathrm{Zn}, \mathrm{Fe}$, and $\mathrm{Mn}$ in 'Manila Cotaxtla 2'; and previous to harvest, 'Tommy Atkins' had greater concentrations of $\mathrm{K}$ and $\mathrm{Zn}$, 'Ataulfo' in $\mathrm{Ca}, \mathrm{Mg}$ and $\mathrm{Cu}$, and 'Manila Cotaxtla 2' showed higher concentration of N (Peralta-Antonio et al., 2015).

Nutrient concentrations in mango fruit have been addressed in other studies. In mango 'Bocado' from three locations of the Cojedes State in Venezuela, different concentrations in the mesocarp were reported

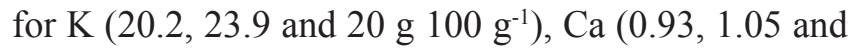

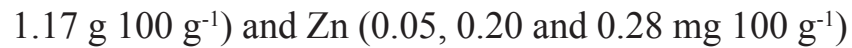
(Milagros-Garrido et al., 2013). In Veracruz, México, the 'Manila' mango grown on a Vertisol soil showed differences for nutrient concentrations among fruit tissues. The highest concentration of $\mathrm{Mg}, \mathrm{Fe}$ and $\mathrm{Mn}$ was in the skin, $\mathrm{K}$ in the mesocarp, Ca in the endocarp, and $\mathrm{N}, \mathrm{P}, \mathrm{Cu}$ and $\mathrm{Zn}$ in the seed (Guzmán-Estrada et al., 1997).

Mango harvest implies the removal of nutrients from the soil. If these nutrients are not returned to the soil, fertility and productivity of the orchard gradually diminish. For efficient management of mango nutrition, it is important to know the amount of minerals removed from the soil by fruit harvest.

In Mexico, there are few studies on nutrient removal by mango harvest. In Apatzingan, Michoacan, the following amounts of nutrients were removed from the soil by fruit of 'Haden' and 'Tommy Atkins', respectively: $\mathrm{N}$ (1.03 and $\left.1.11 \mathrm{~kg} \mathrm{t}^{-1}\right), \mathrm{P}(0.22$ and $\left.0.24 \mathrm{~kg} \mathrm{t}^{-1}\right), \mathrm{K}\left(2.14\right.$ and $\left.1.88 \mathrm{~kg} \mathrm{t}^{-1}\right), \mathrm{Ca}(0.31$ and $\left.0.21 \mathrm{~kg} \mathrm{t}^{-1}\right), \mathrm{Mg}\left(0.14\right.$ and $\left.0.15 \mathrm{~kg} \mathrm{t}^{-1}\right), \mathrm{S}(0.33$ and $\left.0.28 \mathrm{~kg} \mathrm{t}^{-1}\right), \mathrm{Fe}\left(3.8\right.$ and $\left.3.5 \mathrm{~g} \mathrm{t}^{-1}\right), \mathrm{Cu}\left(1.1\right.$ and $\left.1.0 \mathrm{~g} \mathrm{t}^{-1}\right)$, $\mathrm{Mn}\left(4.8\right.$ and $\left.3.2 \mathrm{~g} \mathrm{t}^{-1}\right), \mathrm{Zn}\left(2.8\right.$ and $\left.2.0 \mathrm{gt}^{-1}\right)$, and $\mathrm{B}(1.5$ and $1.6 \mathrm{~g} \mathrm{t}^{-1}$ ) (Mellado-Vázquez et al., 2012). In Cotaxtla, Veracruz, 'Manila' mango fruit harvest removed from the soil the following amounts of nutrients: $\mathrm{N} 1.2 \mathrm{~kg} \mathrm{t}^{-1}$, P $0.2 \mathrm{~kg} \mathrm{t}^{-1}, \mathrm{~K} 2.0 \mathrm{~kg} \mathrm{t}^{-1}$, Ca $0.2 \mathrm{~kg} \mathrm{t}^{-1}, \mathrm{Mg} 0.2 \mathrm{~kg} \mathrm{t}^{-1}$, Fe $5.4 \mathrm{~g} \mathrm{t}^{-1}, \mathrm{Cu} 1.4 \mathrm{~g} \mathrm{t}^{-1}, \mathrm{Mn} 0.4 \mathrm{~g} \mathrm{t}^{-1}$, and $\mathrm{Zn} 2.1 \mathrm{~g} \mathrm{t}^{-1}$ (Guzmán-Estrada et al., 1997). Similar amounts of $\mathrm{N}$, $\mathrm{P}, \mathrm{K}, \mathrm{Ca}, \mathrm{Mg}$, and $\mathrm{S}$ were removed by cv. Zihuaman in China (Xiuchong et al., 2001), 'Kensington Pride' in Australia (Huett and Dirou, 2000), and 'Tommy Atkins' and 'Keitt' in Costa Rica (Fallas et al., 2010). 
Given the economic importance of mango in Mexico, it is necessary to fertilize efficiently to maximize profits and produce high quality fruit for the export market. The objective of this research was to survey mineral concentration in fruit tissues and calculate nutrient removal by fruit harvest of the most important mango cultivars from several production regions of Mexico.

\section{MATERIALS AND METHODS}

Orchard characteristics. In 2008, 15 to 20-year old healthy trees from commercial orchards of four mango cultivars were selected. Cultivars and locations included 'Ataulfo' in the states of Chiapas, Nayarit, and Oaxaca; 'Kent' in the states of Nayarit and Sinaloa; and 'Tommy Atkins' in Nayarit and Campeche. In Nayarit, three orchards per cultivar were used, and in the remaining states, two orchards per cultivar. In each orchard, three bearing trees at least $150 \mathrm{~kg}$ of fruit were randomly selected. The cultivar, location, type of climate (García-Amaro, 1988), water management and soil type (INEGI, 2004; IUSS, 2007) for each of the 17 mango orchards are listed on Table 1.

Fertilization management. Orchard 1 ('Ataulfo' in Oaxaca): Each tree received $6 \mathrm{~kg}$ of 17-17-17 [nitrogen $(\mathrm{N})$, phosphorus $\left(\mathrm{P}_{2} \mathrm{O}_{5}\right)$, potassium $\left.\left(\mathrm{K}_{2} \mathrm{O}\right)\right], 2 \mathrm{~kg}$ urea $(46 \% \mathrm{~N})$ and three foliar sprays of $4 \%$ potassium nitrate $\left(\mathrm{KNO}_{3}\right)$; Orchard 2 ('Ataulfo' in Chiapas): $1.5 \mathrm{~kg}$ of 17-17-17 per tree; Orchard 3 ('Ataulfo' in Chiapas): $1.5 \mathrm{~kg}$ of 17-17-17 per tree; Orchard 4 ('Ataulfo' in Oaxaca): $8 \mathrm{~kg}$ of $17-17-17$ per tree and two foliar sprays of $3 \% \mathrm{KNO}_{3}$; Orchard 5 ('Ataulfo' in Nayarit): trees were not fertilized; Orchard 6 ('Ataulfo' in Nayarit): trees were not fertilized; Orchard 7 ('Ataulfo' in Nayarit): 0.9 kg 17-17-17 per tree; Orchard 8 ('Kent' in Sinaloa): trees received $\left(\mathrm{kg}\right.$ tree $\left.^{-1}\right) 2.8$ ammonium nitrate, 0.15 triple superphosphate, 0.36 potassium sulphate and 0.23 magnesium sulphate; Orchard 9 ('Kent' in Nayarit): trees were not fertilized; Orchard 10 ('Kent' in Nayarit): $0.9 \mathrm{~kg}$ 17-17-17 per tree; Orchard 12 ('Kent' in Sinaloa), Orchard 14 ('Kent' in Nayarit), Orchard 13 ('Tommy Atkins' in Campeche), Orchard 14 ('Tommy Atkins' in Campeche), and

Table 1. Characteristics of mango orchards.

\begin{tabular}{|c|c|c|c|c|c|c|c|c|}
\hline Cultivar & Climate $^{\dagger}$ & $\begin{array}{l}\text { Moisture } \\
\text { regime }\end{array}$ & Soil type & State/Municipality & \multicolumn{2}{|c|}{$\begin{array}{l}\text { Annual rainfall } \\
\text { and months }\end{array}$} & Irrigations ${ }^{\S}$ & $\begin{array}{l}\text { Water } \\
\text { applied }\end{array}$ \\
\hline & & & & \multicolumn{4}{|c|}{$\mathrm{mm}$} & $\mathrm{m}^{3} \mathrm{ha}^{-1}$ \\
\hline Ataulfo & Subw & Irr & Eutric Cambisol & Oaxaca/Tututepec & 1392 & June-Oct. & 17 & 772 \\
\hline Ataulfo & Subw & Irr-1 & Eutric Cambisol & Chiapas/Tapachula & 2037 & May-Oct. & 37 & 5661 \\
\hline Ataulfo & Subw & Irr-2 & Haplic Phaeozem & Chiapas/Huehuetan & 2037 & May- Oct. & 9 & 2830 \\
\hline Ataulfo & Subw & Irr-1 & Eutric Regosol & Oaxaca/Tututepec & 1392 & June-Oct. & 17 & 772 \\
\hline Ataulfo & Subw & $\mathrm{Rf}$ & Eutric Cambisol & Nayarit/Acaponeta & 1350 & June-Sept. & - & - \\
\hline Ataulfo & Subw & $\mathrm{Rf}$ & Eutric Fluvisol & Nayarit/Santiago & 1350 & June- Sept. & - & - \\
\hline Ataulfo & Subw & $\mathrm{Rf}$ & Cromic Luvisol & Nayarit/Tepic & 1350 & June- Sept. & - & - \\
\hline Kent & Subw & Irr-3 & Luvic Phaeozem & Sinaloa/Rosario & 853 & July-Sept. & 40 & 450 \\
\hline Kent & Subw & $\mathrm{Rf}$ & Humic Acrisol & Nayarit/San Blas & 1350 & June- Oct. & - & - \\
\hline Kent & Subw & $\mathrm{Rf}$ & Eutric Cambisol & Nayarit/Acaponeta & 1350 & June-Sept. & - & - \\
\hline Kent & Subw & $\mathrm{Rf}$ & Luvic Phaeozem & Sinaloa/Rosario & 853 & July-Sept. & - & - \\
\hline Kent & Subw & $\mathrm{Rf}$ & Eutric Cambisol & Nayarit/Acaponeta & 1350 & June-Sept. & - & - \\
\hline T. Atkins & Subw & Irr-1 & Hortic Solonchak & Campeche/Campeche & 1100 & June- Oct. & 24 & 2603 \\
\hline T. Atkins & Subw & Irr-1 & Hortic Solonchak & Campeche/Campeche & 1100 & June- Oct. & 24 & 2603 \\
\hline T. Atkins & Subw & Rf & Humic Acrisol & Nayarit/San Blas & 1350 & June- Oct. & - & - \\
\hline T. Atkins & Subw & $\mathrm{Rf}$ & Eutric Cambisol & Nayarit/Acaponeta & 1350 & June-Sept. & - & - \\
\hline T. Atkins & Subw & $\mathrm{Rf}$ & Eutric Cambisol & Nayarit/Compostela & 1548 & June-Oct. & - & - \\
\hline
\end{tabular}

${ }^{\dagger}$ Subw (subhumid warm); Sw (semiarid warm). ${ }^{\ddagger}$ Irr (irrigated); Rf (rainfed); Irr-1 = minisprinkler; Irr-2 = furrow; Irr-3 = drip. ${ }^{\S}$ From flowering to harvest. 
Orchard 15 ('Tommy Atkins' in Nayarit) were not fertilized; Orchard 16 ('Tommy Atkins' in Nayarit): 0.9 kg 17-17-17 per tree; Orchard 17 ('Tommy Atkins' in Nayarit): trees were not fertilized.

Fruit harvesting and processing. In 2009 and 2010, four fruit at physiological maturity were picked from each of the three experimental trees on each orchard. Sampling dates were: Chiapas and Oaxaca, April 2010; Campeche, May 2009; Nayarit, Jun 2009, and Sinaloa, July-August 2009. Harvest criterion was based on fruit size, shape, and color of fruit skin, cavity formation at the pedicel base, and visible lenticel size (Sergent, 1999). At harvest, fresh fruit weight was recorded and fruit washed with tap water and double rinsed with distilled water. Each fruit was separated into skin, mesocarp, endocarp, and seed, and their fresh weight recorded. Tissues were cut in thin slices and dried in a forced air oven (Lab line 34887 Thomas Scientific, Madison, WI, USA) at $70{ }^{\circ} \mathrm{C}$, until constant weight. Samples were then ground in a Wiley mill (3383-L10, Thomas Scientific, Swedesboro, NJ, USA) with a 40 mesh.

Mineral analysis. A composite sample of skin, mesocarp, endocarp or seed tissues was made from each of the four fruit collected per tree. Three replicate samples per tissue (from four fruit per tree) per orchard were analyzed. Total $\mathrm{N}$ was determined by semimicroKjeldahl digestion, modified to include nitrates $\left(\mathrm{NO}_{3}\right)$ (Bremner, 1965). P, Ca, $\mathrm{Mg}, \mathrm{S}, \mathrm{Fe}, \mathrm{Cu}, \mathrm{Mn}$, and $\mathrm{Zn}$ were extracted by wet digestion with a mixture of $\mathrm{HNO}_{3}$ and $\mathrm{HClO}_{4}$ (Jones and Case, 1990), and $\mathrm{K}$ was extracted in water (AOAC, 1990). Except for P, minerals were determined by atomic absorption, using an ICE 3000 spectrometer (Thermo Scientific, Madison, Wisconsin, USA) (AOAC, 1990). P was quantified by ascorbic acid method, and $\mathrm{B}$ was determined by calcination, using the spectrophotometric method of Azometin-H (Enríquez-Reyes, 1989) using a Genesis 20 spectrophotometer (Thermo Scientific, Madison, WI, USA).

Nutrient removal. Nutrient removal per ton of fresh fruit was calculated by the formula, exemplified for nitrogen $(\mathrm{N})$ : , where $\mathrm{NC}_{\mathrm{s}}=$ concentration of $\mathrm{N}$ in skin, $\mathrm{DW}_{\mathrm{s}}=$ skin dry weight, $\mathrm{NC}_{\mathrm{p}}=$ concentration of $\mathrm{N}$ in mesocarp, $\mathrm{DW}_{\mathrm{p}}=$ mesocarp dry weight, $\mathrm{NC}_{\mathrm{h}}=$ concentration of $\mathrm{N}$ in endocarp, $\mathrm{DW}_{\mathrm{h}}=$ endocarp dry weight, $\mathrm{NC}_{\mathrm{e}}=$ concentration of $\mathrm{N}$ in seed, $\mathrm{DW}_{\mathrm{e}}=$ seed dry weight, $\mathrm{F}_{\mathrm{t}}=$ number of fruit in one ton (obtained from the quotient $1000 \mathrm{~kg}$ divided by the fresh weight of the whole fruit; Mellado-Vázquez et al., 2012). The removal of macro- and micronutrients was expressed in $\mathrm{kg} \times \mathrm{t}^{-1}$ and $\mathrm{g} \times \mathrm{t}^{-1}$ of fresh fruit, respectively.

Statistical analysis. A completely randomized design was used with three replications (trees) per orchard; each replication consisted of four fruit per tree. Analysis of variance were conducted using the GLM procedure (SAS for Windows V9). Mean separation was performed with the Waller-Duncan's test $(P \leq 0.05)$.

\section{RESULTS AND DISCUSSION}

\section{Mineral Concentration}

Differences among tissues of mango cultivars. Overall, tissue nutrient concentration was higher in tissues of 'Ataulfo' and 'Tommy Atkins' than in 'Kent'. Mango cultivars showed significant differences in mineral concentration on almost all fruit tissues (Table 2). N, K, Mg and $\mathrm{Zn}$ showed significant concentration differences in at least three of the tissues studied. The concentration of $\mathrm{P}, \mathrm{Ca}$ and $\mathrm{B}$ was significantly different in at least two tissues and the concentration of S and Mn showed no significant differences among the tissues studied.

Differences between orchards of different production regions. For 'Ataulfo' the Oaxaca region seemed to be the most fertile one and skin, mesocarp and seed exhibited higher nutrient concentration in this region than in others (Table 3 ), this could be due to the Oaxaca orchards received more fertilizer than the ones in Chiapas or Nayarit. It is noteworthy that the concentration of $\mathrm{Mn}$ and $\mathrm{Zn}$ was significantly higher in fruit tissues from trees grown in Nayarit, whereas mesocarp tissue from trees in the Nayarit region showed significantly lower concentrations of $\mathrm{K}, \mathrm{Ca}$, $\mathrm{Mg}, \mathrm{S}$ and $\mathrm{Cu}$.

For 'Tommy Atkins', production regions had a significant effect on the nutrient concentration on fruit tissues. Overall, higher nutrient concentrations were found in fruit tissues from Campeche than from Nayarit (Table 4). However, this behavior is not related to the fertilization, since the orchards of Campeche were not fertilized, but can be related to irrigation and in situ fertility. Soil moisture is important for adequate mineral movement in the soil and uptake by roots. A study with 'Dashehari' mango showed more mineral availability at $45 \%$ soil moisture but almost nil at $30 \%$ (Bhriguvanshi et al., 2014). 
Table 2. Mineral concentration in fruit tissues of tree mango cultivars (data were analyzed as a pool of all orchards per cultivar).

\begin{tabular}{|c|c|c|c|c|c|c|c|}
\hline Tissue & Cultivar & $\mathrm{N}$ & $\mathrm{P}$ & $\mathrm{K}$ & $\mathrm{Ca}$ & $\mathrm{Mg}$ & $\mathrm{S}$ \\
\hline & & $\ldots$ & - - - - & $\ldots-\mathrm{g}$ & - - - - & $\ldots$ & - - - \\
\hline \multirow[t]{4}{*}{ Skin } & Ataulfo & $0.5 \mathrm{a}^{\dagger}$ & $0.08 \mathrm{a}$ & $1.1 \mathrm{a}$ & $0.3 \mathrm{~b}$ & $0.15 \mathrm{a}$ & $0.09 \mathrm{a}$ \\
\hline & Kent & $0.4 \mathrm{~b}$ & $0.05 \mathrm{~b}$ & $0.7 \mathrm{~b}$ & $0.3 \mathrm{~b}$ & $0.07 \mathrm{~b}$ & $0.07 \mathrm{a}$ \\
\hline & T. Atkins & $0.5 \mathrm{a}$ & $0.06 \mathrm{ab}$ & $0.7 \mathrm{~b}$ & $0.4 \mathrm{a}$ & $0.08 \mathrm{~b}$ & $0.08 \mathrm{a}$ \\
\hline & $\operatorname{Pr}>F$ & 0.000 & 0.052 & 0.001 & 0.001 & 0.001 & 0.54 \\
\hline \multirow[t]{4}{*}{ Mesocarp } & Ataulfo & $0.5 \mathrm{a}$ & $0.10 \mathrm{a}$ & $1.0 \mathrm{a}$ & $0.1 \mathrm{ab}$ & $0.06 \mathrm{a}$ & $0.09 \mathrm{a}$ \\
\hline & Kent & $0.4 \mathrm{~b}$ & $0.04 \mathrm{~b}$ & $0.5 \mathrm{~b}$ & $0.05 \mathrm{~b}$ & $0.03 \mathrm{~b}$ & $0.06 \mathrm{a}$ \\
\hline & T. Atkins & $0.4 \mathrm{~b}$ & $0.10 \mathrm{ab}$ & $1.0 \mathrm{a}$ & $0.1 \mathrm{a}$ & $0.03 \mathrm{~b}$ & $0.09 \mathrm{a}$ \\
\hline & $\operatorname{Pr}>\mathrm{F}$ & 0.001 & 0.034 & 0.000 & 0.063 & 0.001 & 0.108 \\
\hline \multirow[t]{4}{*}{ Endocarp } & Ataulfo & $0.3 \mathrm{~b}$ & $0.04 \mathrm{~b}$ & $0.3 \mathrm{~b}$ & $0.1 \mathrm{a}$ & $0.08 \mathrm{a}$ & 0.09 \\
\hline & Kent & $0.3 \mathrm{~b}$ & $0.03 \mathrm{~b}$ & $0.3 \mathrm{~b}$ & $0.1 \mathrm{a}$ & $0.05 \mathrm{~b}$ & 0.06 \\
\hline & T. Atkins & $0.4 \mathrm{a}$ & $0.07 \mathrm{a}$ & $0.6 \mathrm{a}$ & $0.1 \mathrm{a}$ & $0.05 \mathrm{~b}$ & 0.09 \\
\hline & $\operatorname{Pr}>F$ & 0.018 & 0.002 & $<0.0001$ & 0.253 & 0.001 & 0.517 \\
\hline \multirow[t]{4}{*}{ Seed } & Ataulfo & $0.9 \mathrm{ab}$ & $0.15 \mathrm{c}$ & $0.90 \mathrm{ab}$ & $0.16 \mathrm{a}$ & $0.15 \mathrm{a}$ & $0.08 \mathrm{a}$ \\
\hline & Kent & $0.9 \mathrm{a}$ & $0.20 \mathrm{~b}$ & $0.80 \mathrm{~b}$ & $0.10 \mathrm{~b}$ & $0.14 \mathrm{a}$ & $0.08 \mathrm{a}$ \\
\hline & T. Atkins & $0.8 \mathrm{~b}$ & $0.24 \mathrm{a}$ & $1.04 \mathrm{a}$ & $0.10 \mathrm{~b}$ & $0.11 \mathrm{~b}$ & $0.11 \mathrm{a}$ \\
\hline & $\operatorname{Pr}>\mathrm{F}$ & 0.016 & $<0.0001$ & 0.005 & $<0.0001$ & 0.001 & 0.994 \\
\hline \multirow[t]{2}{*}{ Tissue } & Cultivar & $\mathrm{Fe}$ & $\mathrm{Cu}$ & $\mathrm{Mn}$ & $\mathrm{Zn}$ & $\mathrm{B}$ & \\
\hline & & $-\cdots$ & ---- & $\mathrm{g} \mathrm{kg}^{-1}-$ & $-\ldots$ & $-\cdots$ & \\
\hline \multirow[t]{4}{*}{ Skin } & Ataulfo & $92.2 \mathrm{a}$ & $4.7 \mathrm{a}$ & $47.1 \mathrm{a}$ & $8.0 \mathrm{a}$ & $14.3 \mathrm{c}$ & \\
\hline & Kent & $27.0 \mathrm{a}$ & $6.1 \mathrm{a}$ & $41.0 \mathrm{a}$ & $5.0 \mathrm{~b}$ & $23.0 \mathrm{~b}$ & \\
\hline & T. Atkins & $25.3 \mathrm{a}$ & $5.0 \mathrm{a}$ & $46.7 \mathrm{a}$ & $8.1 \mathrm{a}$ & $26.9 \mathrm{a}$ & \\
\hline & $\operatorname{Pr}>\mathrm{F}$ & 0.06 & 0.492 & 0.893 & $<0.0001$ & $<0.0001$ & \\
\hline \multirow[t]{4}{*}{ Mesocarp } & Ataulfo & $26.0 \mathrm{a}$ & $5.0 \mathrm{a}$ & $12.4 \mathrm{a}$ & $8.3 \mathrm{a}$ & $10.3 \mathrm{~b}$ & \\
\hline & Kent & $15.4 \mathrm{~b}$ & $5.0 \mathrm{a}$ & $15.0 \mathrm{a}$ & $4.0 \mathrm{~b}$ & $17.0 \mathrm{a}$ & \\
\hline & T. Atkins & $23.2 \mathrm{ab}$ & $5.0 \mathrm{a}$ & $18.2 \mathrm{a}$ & $7.02 \mathrm{a}$ & $16.3 \mathrm{a}$ & \\
\hline & $\operatorname{Pr}>F$ & 0.044 & 0.792 & 0.282 & 0.001 & 0.016 & \\
\hline \multirow[t]{4}{*}{ Endocarp } & Ataulfo & $412.9 \mathrm{a}$ & $5.6 \mathrm{a}$ & $21.2 \mathrm{a}$ & $16.0 \mathrm{a}$ & $14.1 \mathrm{a}$ & \\
\hline & Kent & $24.0 \mathrm{~b}$ & $4.0 \mathrm{~b}$ & $21.0 \mathrm{a}$ & $7.0 \mathrm{~b}$ & $14.2 \mathrm{a}$ & \\
\hline & T. Atkins & $27.5 \mathrm{~b}$ & $5.4 \mathrm{~b}$ & $22.0 \mathrm{a}$ & $10.3 \mathrm{~b}$ & $16.0 \mathrm{a}$ & \\
\hline & $\operatorname{Pr}>F$ & 0.027 & 0.098 & 0.871 & 0.06 & 0.3 & \\
\hline \multirow[t]{4}{*}{ Seed } & Ataulfo & $209.3 \mathrm{a}$ & $8.2 \mathrm{~b}$ & $27.3 \mathrm{a}$ & $10.2 \mathrm{~b}$ & $18.4 \mathrm{ab}$ & \\
\hline & Kent & 24.3 & $12.0 \mathrm{a}$ & $15.0 \mathrm{a}$ & $15.2 \mathrm{a}$ & $22.1 \mathrm{a}$ & \\
\hline & T. Atkins & $25.1 \mathrm{a}$ & $13.2 \mathrm{a}$ & $20.0 \mathrm{a}$ & $15.4 \mathrm{a}$ & $16.4 \mathrm{ab}$ & \\
\hline & $\operatorname{Pr}>F$ & 0.152 & $<0.0001$ & 0.129 & 0.000 & 0.379 & \\
\hline
\end{tabular}


Table 3. Concentration of minerals in 'Ataulfo' mango fruit tissues from three production regions of Mexico.

\begin{tabular}{|c|c|c|c|c|c|c|c|}
\hline & & $-\ldots$ & $-\cdots$ & $-\ldots-g$ & 1 - - - - & $-\ldots$ & $-\ldots$ \\
\hline \multirow[t]{3}{*}{ Skin } & Chiapas & $0.5 \mathrm{a}^{\dagger}$ & $0.07 \mathrm{a}$ & $1.45 \mathrm{a}$ & $0.2 \mathrm{a}$ & $0.20 \mathrm{a}$ & $0.12 \mathrm{~b}$ \\
\hline & Nayarit & $0.5 \mathrm{a}$ & $0.06 \mathrm{a}$ & $0.64 \mathrm{~b}$ & $0.2 \mathrm{a}$ & $0.08 \mathrm{~b}$ & $0.03 \mathrm{c}$ \\
\hline & Oaxaca & $0.5 \mathrm{a}$ & $0.10 \mathrm{a}$ & $1.37 \mathrm{a}$ & $0.3 \mathrm{a}$ & $0.19 \mathrm{a}$ & $0.15 \mathrm{a}$ \\
\hline \multirow[t]{4}{*}{ Mesocarp } & Chiapas & $0.61 \mathrm{a}$ & $0.06 \mathrm{ab}$ & $1.36 \mathrm{a}$ & $0.08 \mathrm{a}$ & $0.07 \mathrm{a}$ & $0.11 \mathrm{~b}$ \\
\hline & Nayarit & $0.45 \mathrm{~b}$ & $0.05 \mathrm{~b}$ & $0.74 \mathrm{c}$ & $0.04 \mathrm{~b}$ & $0.03 \mathrm{~b}$ & $0.06 \mathrm{c}$ \\
\hline & Oaxaca & $0.56 \mathrm{ab}$ & $0.08 \mathrm{a}$ & $1.12 \mathrm{~b}$ & $0.09 \mathrm{a}$ & $0.07 \mathrm{a}$ & $0.14 \mathrm{a}$ \\
\hline & $\operatorname{Pr}>F$ & 0.042 & 0.026 & 0.000 & $<0.0001$ & $<0.0001$ & $<0.0001$ \\
\hline Endocarp & $\operatorname{Pr}>F$ & $<0.0001$ & 0.005 & $<0.0001$ & 0.1 & 0.001 & $<0.0001$ \\
\hline \multirow[t]{4}{*}{ Seed } & Chiapas & $0.8 \mathrm{~b}$ & $0.10 \mathrm{c}$ & $0.9 \mathrm{~b}$ & $0.15 \mathrm{ab}$ & $0.17 \mathrm{a}$ & $0.11 \mathrm{~b}$ \\
\hline & Nayarit & $0.8 \mathrm{~b}$ & $0.15 b$ & $0.8 \mathrm{c}$ & $0.14 \mathrm{~b}$ & $0.12 \mathrm{~b}$ & $0.02 \mathrm{c}$ \\
\hline & Oaxaca & $1.0 \mathrm{a}$ & $0.18 \mathrm{a}$ & $1.0 \mathrm{a}$ & $0.19 \mathrm{a}$ & $0.14 \mathrm{ab}$ & $0.15 \mathrm{a}$ \\
\hline & $\operatorname{Pr}>F$ & 0.001 & 0.001 & 0.001 & 0.044 & 0.017 & $<0.0001$ \\
\hline \multirow[t]{2}{*}{ Tissue } & Cultivar & $\mathrm{Fe}$ & $\mathrm{Cu}$ & $\mathrm{Mn}$ & $\mathrm{Zn}$ & B & \\
\hline & & $-\ldots-$. & $-\ldots$ & $\mathrm{mg} \mathrm{kg}^{-1}-$ & 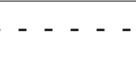 & - - - - - & \\
\hline Skin & Chiapas & $13.0 \mathrm{~b}$ & $5.9 \mathrm{a}$ & $20.0 \mathrm{~b}$ & $7.3 \mathrm{a}$ & $15.3 \mathrm{a}$ & \\
\hline \multirow{2}{*}{ Mesocarp } & Oaxaca & $23.8 \mathrm{~b}$ & $5.1 \mathrm{~b}$ & $3.1 \mathrm{~b}$ & $9.0 \mathrm{ab}$ & $6.9 \mathrm{~b}$ & \\
\hline & $\operatorname{Pr}>F$ & 0.013 & 0.002 & 0.02 & 0.006 & $<0.0001$ & \\
\hline \multirow[t]{4}{*}{ Endocarp } & Chiapas & $22.2 \mathrm{~b}$ & $2.9 \mathrm{~b}$ & $10.4 \mathrm{~b}$ & $5.5 \mathrm{~b}$ & $3.8 \mathrm{~b}$ & \\
\hline & Nayarit & $19.2 \mathrm{~b}$ & $4.6 \mathrm{~b}$ & $33.6 \mathrm{a}$ & $25.7 \mathrm{a}$ & $25.7 \mathrm{a}$ & \\
\hline & Oaxaca & $1394.1 \mathrm{a}$ & $9.7 \mathrm{a}$ & $13.2 \mathrm{~b}$ & $11.2 \mathrm{ab}$ & $6.6 \mathrm{~b}$ & \\
\hline & $\operatorname{Pr}>F$ & $<0.0001$ & 0.001 & 0.016 & 0.021 & $<0.0001$ & \\
\hline \multirow[t]{4}{*}{ Seed } & Chiapas & $34.1 \mathrm{~b}$ & $8.0 \mathrm{~b}$ & $15.7 \mathrm{~b}$ & $10.6 \mathrm{a}$ & $12.93 \mathrm{~b}$ & \\
\hline & Nayarit & $14.0 \mathrm{~b}$ & $6.8 \mathrm{~b}$ & $45.0 \mathrm{a}$ & $9.00 \mathrm{a}$ & $26.23 \mathrm{a}$ & \\
\hline & Oaxaca & $677.4 \mathrm{a}$ & $10.5 \mathrm{a}$ & $12.1 \mathrm{~b}$ & $11.7 \mathrm{a}$ & $12.26 \mathrm{~b}$ & \\
\hline & $\operatorname{Pr}>F$ & 0.018 & 0.013 & 0.014 & 0.109 & 0.002 & \\
\hline
\end{tabular}


Table 4. Concentration of minerals in 'Tommy Atkins' mango fruit tissues from two production regions of Mexico.

\begin{tabular}{|c|c|c|c|c|c|c|c|}
\hline Tissue & Cultivar & $\mathrm{N}$ & $\mathrm{P}$ & $\mathrm{K}$ & $\mathrm{Ca}$ & $\mathrm{Mg}$ & $\mathrm{S}$ \\
\hline & & 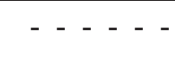 & $-\cdots$ & $-\ldots-g$ & $-\ldots$ & 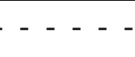 & $-\ldots$ \\
\hline \multirow[t]{3}{*}{ Skin } & Campeche & $0.5 \mathrm{a}^{\dagger}$ & $0.09 \mathrm{a}$ & $1.0 \mathrm{a}$ & $0.4 \mathrm{a}$ & $0.1 \mathrm{a}$ & $0.15 \mathrm{a}$ \\
\hline & Nayarit & $0.4 \mathrm{~b}$ & $0.04 \mathrm{~b}$ & $0.5 \mathrm{~b}$ & $0.3 \mathrm{~b}$ & $0.1 \mathrm{a}$ & $0.03 \mathrm{~b}$ \\
\hline & $\operatorname{Pr}>F$ & 0.027 & 0.002 & 0.000 & 0.031 & 0.112 & $<0.0001$ \\
\hline \multirow[t]{3}{*}{ Mesocarp } & Campeche & $0.4 \mathrm{a}$ & $0.1 \mathrm{a}$ & $1.3 \mathrm{a}$ & $0.13 \mathrm{a}$ & $0.04 \mathrm{a}$ & $0.15 \mathrm{a}$ \\
\hline & Nayarit & $0.4 \mathrm{a}$ & $0.04 \mathrm{~b}$ & $0.6 \mathrm{~b}$ & $0.05 \mathrm{~b}$ & $0.03 \mathrm{a}$ & $0.05 \mathrm{~b}$ \\
\hline & $\operatorname{Pr}>F$ & 0.099 & 0.000 & $<0.0001$ & $<0.0001$ & 0.163 & $<0.0001$ \\
\hline \multirow[t]{3}{*}{ Endocarp } & Campeche & $0.53 \mathrm{a}$ & 0.10 & $1.0 \mathrm{a}$ & 0.23 & 0.05 & $0.15 \mathrm{a}$ \\
\hline & Nayarit & $0.37 \mathrm{~b}$ & 0.10 & $0.4 \mathrm{~b}$ & 0.17 & 0.07 & $0.02 \mathrm{~b}$ \\
\hline & $\operatorname{Pr}>F$ & 0.009 & 0.28 & 0.000 & 0.279 & 0.07 & $<0.0001$ \\
\hline \multirow[t]{3}{*}{ Seed } & Campeche & $0.7 \mathrm{~b}$ & $0.2 \mathrm{a}$ & $1.5 \mathrm{a}$ & $0.14 \mathrm{a}$ & $0.09 \mathrm{a}$ & $0.16 \mathrm{a}$ \\
\hline & Nayarit & $0.8 \mathrm{a}$ & $0.2 \mathrm{a}$ & $1.0 \mathrm{~b}$ & $0.06 \mathrm{~b}$ & $0.11 \mathrm{a}$ & $0.04 \mathrm{~b}$ \\
\hline & $\operatorname{Pr}>F$ & 0.013 & 0.491 & $<0.0001$ & 0.004 & 0.073 & $<0.0001$ \\
\hline \multirow[t]{2}{*}{ Tissue } & Cultivar & $\mathrm{Fe}$ & $\mathrm{Cu}$ & $\mathrm{Mn}$ & $\mathrm{Zn}$ & $\mathrm{B}$ & \\
\hline & & $-\ldots$ & - - - & $\mathrm{mg} \mathrm{kg}^{-1}-$ & - - - - & - . - - & \\
\hline \multirow[t]{3}{*}{ Skin } & Campeche & $33.9 \mathrm{a}$ & $5.8 \mathrm{a}$ & $22.9 \mathrm{a}$ & $11.1 \mathrm{a}$ & $22.4 \mathrm{a}$ & \\
\hline & Nayarit & $19.6 \mathrm{~b}$ & $4.4 \mathrm{~b}$ & $62.6 \mathrm{a}$ & $6.0 \mathrm{~b}$ & $29.7 \mathrm{a}$ & \\
\hline & $\operatorname{Pr}>F$ & 0.000 & 0.045 & 0.061 & $<0.0001$ & 0.098 & \\
\hline \multirow[t]{3}{*}{ Mesocarp } & Campeche & $35.4 \mathrm{a}$ & $5.7 \mathrm{a}$ & $19.5 \mathrm{a}$ & $11.1 \mathrm{a}$ & $8.0 \mathrm{~b}$ & \\
\hline & Nayarit & $15.0 \mathrm{~b}$ & $5.0 \mathrm{~b}$ & $19.6 \mathrm{a}$ & $4.3 \mathrm{~b}$ & $21.9 \mathrm{a}$ & \\
\hline & $\operatorname{Pr}>F$ & 0.002 & 0.089 & 0.995 & $<0.0001$ & 0.002 & \\
\hline \multirow[t]{3}{*}{ Endocarp } & Campeche & $53.0 \mathrm{a}$ & 6.40 & 22.00 & 12.4 & $25.0 \mathrm{a}$ & \\
\hline & Nayarit & $11.0 \mathrm{~b}$ & 5.00 & 25.80 & 9.00 & $14.0 \mathrm{~b}$ & \\
\hline & $\operatorname{Pr}>F$ & $<0.0001$ & 0.104 & 0.655 & 0.136 & $<0.0001$ & \\
\hline \multirow[t]{3}{*}{ Seed } & Campeche & $50.1 \mathrm{a}$ & $13.1 \mathrm{a}$ & $24.0 \mathrm{a}$ & $18.1 \mathrm{a}$ & $17.7 \mathrm{a}$ & \\
\hline & Nayarit & $12.0 \mathrm{~b}$ & $13.2 \mathrm{a}$ & $17.0 \mathrm{a}$ & $12.5 \mathrm{~b}$ & $18.8 \mathrm{a}$ & \\
\hline & $\operatorname{Pr}>F$ & $<0.0001$ & 0.911 & 0.100 & 0.002 & 0.731 & \\
\hline
\end{tabular}

${ }^{\dagger}$ Means with different letter in the same column within each tissue are statistically different (Waller-Duncan’s test, $P \leq 0.05$ ).

In 'Kent', fruit from Sinaloa had significantly higher concentrations of $\mathrm{P}, \mathrm{Mg}$ and $\mathrm{S}$ in the skin, $\mathrm{S}$ in the mesocarp, $\mathrm{P}, \mathrm{Mg}, \mathrm{S}, \mathrm{Fe}, \mathrm{Cu}$ and $\mathrm{Mn}$ in the endocarp, and $\mathrm{S}$ in the seed than from Nayarit. 'Kent' fruit from Nayarit had greater concentrations of $\mathrm{Mn}$ in the skin and $\mathrm{P}, \mathrm{Mg}, \mathrm{Cu}$, and $\mathrm{Mn}$ in the endocarp, as compared to fruit from the Sinaloa region (Table 5). The greatest concentration of nutrients in the fruit tissues of Sinaloa can be attributed to the fact that one of the orchards
(Orchard 8; as described in the fertilization management section)) received greater fertilization than that applied to the orchard 10 of Nayarit, in addition to the fact that the Sinaloa orchard 8 had irrigation.

Overall, mineral concentration in fruit tissues were affected by production region and mango cultivar. Mineral concentrations in fruit tissues of 'Ataulfo' and 'Tommy Atkins' were more affected by production region than in 'Kent'. Similar results were found for 
'Tommy Atkins' in Venezuela, where fruit from this cultivar had higher concentrations of $\mathrm{N}, \mathrm{P}$, and $\mathrm{Mg}$ than 'Haden' and 'Kent' (Laborem-Escalona et al., 1979). In this study, the concentration of $\mathrm{N}, \mathrm{P}, \mathrm{K}, \mathrm{Ca}$ and $\mathrm{Mg}$ in the mesocarp and seed of 'Ataulfo' were similar to that reported for 'Manila' mango in Veracruz, Mexico (Guzmán-Estrada et al., 1997) and 'Sensation' in Nelspruit, South Africa (Stassen et al., 2000).

In other fruit trees, such as avocado, the effect of climate on fruit mineral composition has been reported. In Michoacán, 'Hass' avocado fruit in a subhumid semi-warm climate presented the highest $\mathrm{P}, \mathrm{Fe}$ and $\mathrm{B}$ contents in the skin, $\mathrm{Fe}$ and $\mathrm{Cu}$ in the testa and $\mathrm{B}$ in the seed; while in the humid semiwarm climate the highest concentrations of $\mathrm{Ca}$ in skin and $\mathrm{K}$ and $\mathrm{Zn}$ in testa were present. However, in the subhumid temperate climate there were the highest concentrations of $\mathrm{N}$ and $\mathrm{Mn}$ in skin, $\mathrm{N}, \mathrm{K}$ and $\mathrm{Ca}$ in mesocarp and $\mathrm{Mg}, \mathrm{S}$ and $\mathrm{Mn}$ in the testa (SalazarGarcía et al., 2011).

Table 5. Concentration of minerals in 'Kent' mango fruit tissues from two production regions of Mexico.

\begin{tabular}{|c|c|c|c|c|c|c|c|}
\hline Tissue & Cultivar & $\mathrm{N}$ & $\mathrm{P}$ & $\mathrm{K}$ & $\mathrm{Ca}$ & $\mathrm{Mg}$ & $\mathrm{S}$ \\
\hline & & - - - & $\cdots$ & $-\cdots$ & $\ldots$ & $\ldots$ & - - - - \\
\hline \multirow[t]{3}{*}{ Skin } & Nayarit & $0.3 \mathrm{a}^{\dagger}$ & $0.03 \mathrm{~b}$ & $0.6 \mathrm{a}$ & $0.2 \mathrm{a}$ & $0.02 \mathrm{~b}$ & $0.03 \mathrm{~b}$ \\
\hline & Sinaloa & $0.4 \mathrm{a}$ & $0.07 \mathrm{a}$ & $0.7 \mathrm{a}$ & $0.2 \mathrm{a}$ & $0.14 \mathrm{a}$ & $0.12 \mathrm{a}$ \\
\hline & $\operatorname{Pr}>F$ & 0.394 & 0.002 & 0.219 & 0.415 & $<0.0001$ & $<0.0001$ \\
\hline \multirow[t]{3}{*}{ Mesocarp } & Nayarit & $0.3 \mathrm{a}$ & $0.03 \mathrm{a}$ & $0.5 \mathrm{a}$ & $0.05 \mathrm{a}$ & $0.03 \mathrm{a}$ & $0.04 \mathrm{~b}$ \\
\hline & Sinaloa & $0.4 \mathrm{a}$ & $0.05 \mathrm{a}$ & $0.5 \mathrm{a}$ & $0.06 \mathrm{a}$ & $0.04 \mathrm{a}$ & $0.09 \mathrm{a}$ \\
\hline & $\operatorname{Pr}>F$ & 0.224 & 0.073 & 0.637 & 0.201 & 0.194 & 0.014 \\
\hline \multirow[t]{3}{*}{ Endocarp } & Nayarit & $0.3 \mathrm{a}$ & $0.03 \mathrm{a}$ & $0.3 \mathrm{a}$ & $0.2 \mathrm{a}$ & $0.06 \mathrm{a}$ & $0.02 \mathrm{~b}$ \\
\hline & Sinaloa & $0.2 \mathrm{a}$ & $0.02 \mathrm{~b}$ & $0.2 \mathrm{a}$ & $0.1 \mathrm{a}$ & $0.03 \mathrm{~b}$ & $0.12 \mathrm{a}$ \\
\hline & $\operatorname{Pr}>\mathrm{F}$ & 0.36 & 0.02 & 0.46 & 0.06 & 0.003 & $<0.0001$ \\
\hline \multirow[t]{3}{*}{ Seed } & Nayarit & $0.9 \mathrm{a}$ & $0.1 \mathrm{a}$ & $0.7 \mathrm{a}$ & $0.09 \mathrm{a}$ & $0.12 \mathrm{a}$ & $0.06 \mathrm{~b}$ \\
\hline & Sinaloa & $0.9 \mathrm{a}$ & $0.2 \mathrm{a}$ & $0.8 \mathrm{a}$ & $0.08 \mathrm{a}$ & $0.15 \mathrm{a}$ & $0.13 \mathrm{a}$ \\
\hline & $\operatorname{Pr}>F$ & 0.074 & 0.253 & 0.236 & 0.813 & 0.112 & 0.000 \\
\hline \multirow[t]{2}{*}{ Tissue } & Cultivar & $\mathrm{Fe}$ & $\mathrm{Cu}$ & $\mathrm{Mn}$ & $\mathrm{Zn}$ & B & \\
\hline & & $-\cdots$ & $\cdots$ & $\mathrm{g} \mathrm{kg}^{-1}-$ & $\cdots$ & - - - & \\
\hline \multirow[t]{3}{*}{ Skin } & Nayarit & $18.1 \mathrm{a}$ & $7.9 \mathrm{a}$ & $57.7 \mathrm{a}$ & $4.6 \mathrm{a}$ & $24.3 \mathrm{a}$ & \\
\hline & Sinaloa & $39.9 \mathrm{a}$ & $3.2 \mathrm{a}$ & $14.7 \mathrm{~b}$ & $5.2 \mathrm{a}$ & $20.0 \mathrm{a}$ & \\
\hline & $\operatorname{Pr}>F$ & 0.083 & 0.115 & 0.018 & 0.329 & 0.187 & \\
\hline \multirow[t]{3}{*}{ Mesocarp } & Nayarit & $14.4 \mathrm{a}$ & $4.9 \mathrm{a}$ & $18.5 \mathrm{a}$ & $3.4 \mathrm{a}$ & $18.6 \mathrm{a}$ & \\
\hline & Sinaloa & $16.9 \mathrm{a}$ & $4.6 \mathrm{a}$ & $8.6 \mathrm{a}$ & $4.5 \mathrm{a}$ & $13.5 \mathrm{a}$ & \\
\hline & $\operatorname{Pr}>F$ & 0.677 & 0.578 & 0.146 & 0.137 & 0.134 & \\
\hline \multirow[t]{3}{*}{ Endocarp } & Nayarit & $10.2 \mathrm{~b}$ & $4.2 \mathrm{a}$ & $30.8 \mathrm{a}$ & $8.7 \mathrm{a}$ & $14.9 \mathrm{a}$ & \\
\hline & Sinaloa & $44.2 \mathrm{a}$ & $3.2 \mathrm{~b}$ & $5.4 \mathrm{~b}$ & $4.4 \mathrm{a}$ & $13.1 \mathrm{a}$ & \\
\hline & $\operatorname{Pr}>F$ & 0.001 & 0.002 & 0.042 & 0.285 & 0.382 & \\
\hline \multirow[t]{3}{*}{ Seed } & Nayarit & $18.4 \mathrm{a}$ & $13.2 \mathrm{a}$ & $19.3 \mathrm{a}$ & $14.7 \mathrm{a}$ & $24.6 \mathrm{a}$ & \\
\hline & Sinaloa & $32.9 \mathrm{a}$ & 9.9 a & $7.7 \mathrm{a}$ & $15.9 \mathrm{a}$ & $18.2 \mathrm{a}$ & \\
\hline & $\operatorname{Pr}>F$ & 0.056 & 0.138 & 0.054 & 0.625 & 0.165 & \\
\hline
\end{tabular}


In general, 'Ataulfo' fruit from the Chiapas and Oaxaca regions had greater concentration of minerals elements, than fruit from Nayarit. This could be the result of fertilization practices, as in Chiapas and Oaxaca the orchards were fertilized, while in Nayarit, where around $60 \%$ of mango growers fertilize their orchards (Pérez-Barraza et al., 2007), only one of the three orchards received fertilization. The differences in the removal of $\mathrm{K}, \mathrm{Ca}, \mathrm{Mg}$ and $\mathrm{S}$, accentuate the need to have a specific nutrition management program for each orchard. Another factor to consider is that in Nayarit, the water available for the trees come from the rainfall. Considering that the rainy season goes from the beginning of June to the middle of October, this may have been insufficient for the assimilation of soil nutrients during the dry season, since the lower availability of moisture in the rainfed orchards could have limited this process $(\mathrm{Hu}$ and Schmidhalter, 2005). Other factors that could influence are the environmental conditions that affect root growth, such as soil compaction, water scarcity, insufficient soil aeration, extreme soil temperatures, as well as shortage or excess nutrients in the soil (Kafkafi, 2008).

'Tommy Atkins' orchards from Campeche received irrigation and fertilization. This may explain a higher mineral concentration in their fruit as compared to fruit from Nayarit. Soil moisture is important for adequate mineral movement in the soil and uptake by roots. A study with 'Dashehari' mango showed more mineral availability at $45 \%$ soil moisture but almost nil at 30\% (Bhriguvanshi et al., 2014).

'Kent' fruit tissues showed some differences in mineral concentrations. Soil fertility and fertilizer applied (magnesium sulphate) in the orchard 8 of Sinaloa could explain the high concentration of $\mathrm{Mg}$ in skin, and endocarp tissues.

\section{Nutrient Removal}

'Ataulfo'. Total nutrient removal in 'Ataulfo' was significantly affected by the production region. Fruit from Oaxaca removed more $\mathrm{P}, \mathrm{Ca}, \mathrm{S}$ and $\mathrm{Fe}$ and $\mathrm{Cu}$ than from other regions. In Chiapas and Oaxaca, fruit removed more $\mathrm{K}$ and $\mathrm{Mg}$.
'Tommy Atkins'. Total removal of P, K, Ca, and S was greater at Campeche than at Nayarit. In the case of B, fruit from Nayarit had more than twice the amount removed by fruit from Campeche.

'Kent'. Producing region had a significant effect on total removal of $\mathrm{P}, \mathrm{Mg}$, and $\mathrm{S}$ (Table 6) with more nutrient removal by fruit occurring in Sinaloa than in Nayarit.

In the present research, total removal of nutrients by fruit did not vary significantly among cultivars (Table 6). Exceptions were $\mathrm{K}, \mathrm{Mg}$ and $\mathrm{B}$. As for $\mathrm{K}$ and $\mathrm{Mg}$ they were removed in higher amounts in 'Ataulfo' than in 'Kent' and 'Tommy Atkins; in the case of B, it was removed in greater quantities by the fruit of 'Kent', compared to 'Ataulfo' and 'Tommy Atkins' fruit.

Different production regions of the same mango cultivar had a significant effect on nutrient removal by fruit harvest. 'Ataulfo' removed significantly more $\mathrm{P}, \mathrm{Ca}, \mathrm{S}$, and $\mathrm{Fe}$ in Oaxaca than in Chiapas or Nayarit. In Campeche, the fruit of 'Tommy Atkins' removed more $\mathrm{P}, \mathrm{K}, \mathrm{Ca}$ and $\mathrm{S}$ than the fruit of Nayarit, while in Sinaloa the fruit of 'Kent' removed more P, Mg and S than those of Nayarit. This could have been the result of more fertilization provided in the region. Despite these differences, the greatest removal of $\mathrm{K}, \mathrm{N}, \mathrm{Fe}$, $\mathrm{Mn}$ and $\mathrm{B}$ was common to the three cultivars, so it is considered essential to include their application in the fertilization plan of each orchard as well as their monitoring by plant tissue analysis.

The findings found in this study have implications for the nutritional management of the mango cultivars studied. Some nutrients can be supplied considering only the cultivar; however, for others the production region should be considered. It should be noted that the amount of nutrients removed described in Table 6 is the minimum amount to be restored for each ton of fresh fruit harvested. As for fertilization recommendations other factors should be considered, such as frequency and amount of pruned wood (Cruz-Barrón et al., 2014), leaf and soil analysis, the nutrient requirement of the tree for a given production target, tree phenology, and the efficiency and method of applying fertilizers and/or manures, among other factors (Salazar-García et al., 2014). 
Table 6. Total fruit nutrient removal by different mango cultivars from some production regions of Mexico.

\begin{tabular}{|c|c|c|c|c|c|c|c|}
\hline Cultivar & Region & $\mathrm{N}$ & $\mathrm{P}$ & $\mathrm{K}$ & $\mathrm{Ca}$ & $\mathrm{Mg}$ & $\mathrm{S}$ \\
\hline & & $\ldots$ & $\ldots$ & - - - & - - - & $\ldots$ & - - - \\
\hline \multirow[t]{4}{*}{ Ataulfo } & Chiapas & $1.0 \mathrm{a}^{\dagger}$ & $0.11 \mathrm{~b}$ & $2.1 \mathrm{a}$ & $0.2 \mathrm{~b}$ & $0.2 \mathrm{a}$ & $0.2 \mathrm{~b}$ \\
\hline & Nayarit & $1.0 \mathrm{a}$ & $0.12 \mathrm{~b}$ & $1.3 \mathrm{~b}$ & $0.1 \mathrm{c}$ & $0.1 \mathrm{~b}$ & $0.1 \mathrm{c}$ \\
\hline & Oaxaca & $1.2 \mathrm{a}$ & $0.19 \mathrm{a}$ & $2.2 \mathrm{a}$ & $0.3 \mathrm{a}$ & $0.2 \mathrm{a}$ & $0.3 \mathrm{a}$ \\
\hline & $\operatorname{Pr}>F$ & 0.366 & 0.021 & 0.005 & 0.002 & $<0.0001$ & $<0.0001$ \\
\hline \multirow[t]{3}{*}{ Tommy Atkins } & Campeche & $0.78 \mathrm{a}$ & $0.16 \mathrm{a}$ & $1.9 \mathrm{a}$ & $0.3 \mathrm{a}$ & $0.08 \mathrm{a}$ & $0.25 \mathrm{a}$ \\
\hline & Nayarit & $0.9 \mathrm{a}$ & $0.12 \mathrm{~b}$ & $1.2 \mathrm{~b}$ & $0.2 \mathrm{~b}$ & $0.09 \mathrm{a}$ & $0.08 \mathrm{~b}$ \\
\hline & $\operatorname{Pr}>F$ & 0.446 & 0.001 & 0.001 & 0.031 & 0.408 & $<0.0001$ \\
\hline \multirow[t]{3}{*}{ Kent } & Nayarit & $0.8 \mathrm{a}$ & $0.11 \mathrm{~b}$ & $1.2 \mathrm{a}$ & $0.2 \mathrm{a}$ & $0.08 \mathrm{~b}$ & $0.09 \mathrm{~b}$ \\
\hline & Sinaloa & $1.0 \mathrm{a}$ & $0.17 \mathrm{a}$ & $1.3 \mathrm{a}$ & $0.4 \mathrm{a}$ & $0.15 \mathrm{a}$ & $0.23 \mathrm{a}$ \\
\hline & $\operatorname{Pr}>F$ & 0.272 & 0.003 & 0.427 & 0.089 & 0.001 & $<0.0001$ \\
\hline \multirow[t]{2}{*}{ Cultivar } & Region & $\mathrm{Fe}$ & $\mathrm{Cu}$ & $\mathrm{Mn}$ & $\mathrm{Zn}$ & B & \\
\hline & & $-\ldots$ & - - - & $\mathrm{g} \mathrm{t}^{-1}-$ & - - - & $\ldots$ & \\
\hline \multirow[t]{4}{*}{ Ataulfo } & Chiapas & $4.9 \mathrm{~b}$ & $1.1 \mathrm{ab}$ & $2.3 \mathrm{~b}$ & $1.7 \mathrm{a}$ & $1.6 \mathrm{~b}$ & \\
\hline & Nayarit & $4.1 \mathrm{~b}$ & $0.6 \mathrm{~b}$ & $6.9 \mathrm{a}$ & $1.5 \mathrm{a}$ & $3.2 \mathrm{a}$ & \\
\hline & Oaxaca & $55.1 \mathrm{a}$ & $1.2 \mathrm{a}$ & $1.5 \mathrm{~b}$ & $1.9 \mathrm{a}$ & $1.7 \mathrm{~b}$ & \\
\hline & $\operatorname{Pr}>F$ & 0.001 & 0.027 & 0.007 & 0.361 & $<0.0001$ & \\
\hline \multirow[t]{3}{*}{ Tommy Atkins } & Campeche & $6.3 \mathrm{a}$ & $1.0 \mathrm{a}$ & $3.3 \mathrm{a}$ & $1.9 \mathrm{a}$ & $2.0 \mathrm{~b}$ & \\
\hline & Nayarit & $6.0 \mathrm{a}$ & $1.2 \mathrm{a}$ & $5.6 \mathrm{a}$ & $1.5 \mathrm{a}$ & $4.3 \mathrm{a}$ & \\
\hline & $\operatorname{Pr}>F$ & 0.944 & 0.331 & 0.311 & 0.428 & 0.003 & \\
\hline \multirow[t]{3}{*}{ Kent } & Nayarit & $5.4 \mathrm{a}$ & $1.2 \mathrm{a}$ & $5.8 \mathrm{a}$ & $1.3 \mathrm{a}$ & $4.2 \mathrm{a}$ & \\
\hline & Sinaloa & $7.9 \mathrm{a}$ & $1.1 \mathrm{a}$ & $1.9 \mathrm{a}$ & $1.5 \mathrm{a}$ & $3.2 \mathrm{a}$ & \\
\hline & $\operatorname{Pr}>F$ & 0.497 & 0.334 & 0.09 & 0.688 & 0.135 & \\
\hline
\end{tabular}

${ }^{\top}$ Means with different letter in the same column within each cultivar are statistically different (Waller-Duncan’s test, $P \leq 0.05$ ).

\section{CONCLUSIONS}

A survey of the fruit nutrient elements status of commercially important mango cultivars grown in several production regions of Mexico was made. Differences in the concentration of $\mathrm{N}, \mathrm{K}, \mathrm{Mg}$, and $\mathrm{Zn}$ were found among cultivars and tissues. Concentration of $\mathrm{P}, \mathrm{S}, \mathrm{Cu}$, and $\mathrm{Mn}$ in the skin, $\mathrm{Ca}, \mathrm{Cu}$, and $\mathrm{Mn}$ in the mesocarp, $\mathrm{Ca}, \mathrm{S}, \mathrm{Mn}$, and $\mathrm{B}$ in endocarp, and $\mathrm{S}, \mathrm{Fe}$, and $\mathrm{Mn}$ in the seed were not affected by mango cultivar. Production region affected concentration of minerals in 'Ataulfo' fruit more than in 'Tommy Atkins' and 'Kent'. Nutrient removal by mango fruit tissues was little affected in cvs. Ataulfo, Tommy Atkins and Kent. The regions with the greatest nutrient removal were Oaxaca, Campeche and Sinaloa for 'Ataulfo', 'Tommy Atkins' and 'Kent', respectively. This study provides baseline concentration in fruit tissues and nutrient removal values which can be used by growers, researchers, extension personnel, and mango consultants to implement best management practices for mango fertilization.

\section{ACKNOWLEDGEMENTS}

This research was partially funded by INIFAP and the CONACYT-FORDECYT Program (Project 115830). The authors thank Roberto Gómez-Aguilar (Nayarit), César A. Treviño-De la Fuente (Michoacán), Martín F. Tucuch-Cauich (Campeche), Moisés Alonso- 
Báez (Chiapas), Aristeo Barrios-Ayala (Guerrero), Heidi M. Medina-Montenegro (Sinaloa), Manuel E. Ovando-Cruz (Oaxaca), José González-Valdivia and Sergio O. Álvarez-López (Nayarit), for their assistance on fruit sampling and data processing.

\section{REFERENCES}

AOAC (Association of Official Analytical Chemists). 1990. Official methods of analysis. 15th ed. Arlington, VI, USA. ISBN 0-935584-85-4.

Bhriguvanshi, S. R., T. Adak, K. Kumar, A. Singh, and V. K. Singh. 2014. Impact of varying soil moisture regimes on growth and soil nutrient availability in mango. Indian J. Soil Conserv. 42: 68-73.

Bremner, J. M. 1965. Total Nitrogen. pp. 1049-1178. In: C. A. Black (ed.) Methods of soil analysis. Part 2: Chemical and microbial properties. Number 9 in series Agronomy. American Society of Agronomy, Inc. Publisher. Madison, WI, USA.

Castro-López, M. G., S. Salazar-García, I. J. L. González-Durán, R. Medina-Torres y J. González-Valdivia. 2012. Evolución nutrimental foliar en tres cultivares de mango en Nayarit, México. Rev. Mex. Cienc. Agríc. 3: 685-700.

Cruz-Barrón, V., R. Bugarín-Montoya, G. Alejo-Santiago, G. LunaEsquivel y P. Juárez-López. 2014. Extracción y requerimiento de macronutrimentos en mango 'Ataulfo' (Mangifera indica L.) con manejo de poda anual y bianual. Rev. Mex. Cienc. Agríc. 5: 229-239.

Enríquez-Reyes, S. 1989. Análisis de boro en suelos y plantas mediante el método de Azometina-H. Terra 7: 13-20.

Fallas, R., F. Bertsch, E. Miranda y C. Henríquez. 2010. Análisis de crecimiento y absorción de nutrimentos de frutos de mango, cultivares Tommy Atkins y Keith. Agronomía Costarricense 34: $1-15$.

García-Amaro, E. 1988. Modificaciones al sistema de clasificación climática de Köppen. Instituto de Geografía, UNAM. México, D. F.

Guzmán-Estrada, C., R. Mosqueda-Vázquez, S. Alcalde-Blanco, and A. Martínez-Garza. 1997. Content and extraction of several minerals by mango fruits of Manila cultivar. Acta Hort. 455: 465-470.

Huett, D. O. and J. F. Dirou. 2000. An evaluation of the rationale for fertilizer management of tropical fruit crops. Aust. J. Exp. Agric. 40: 1137-1143. doi: 10.1071/EA00047.

$\mathrm{Hu}, \mathrm{Y}$. and U. Schmidhalter. 2005. Drought and salinity: A comparison of their effects on mineral nutrition of plants. J. Plant Nutr. Soil Sci. 168: 541-549. doi: https://doi.org/10.1002/ jpln.200420516.

INEGI (Instituto Nacional de Estadística y Geografía). 2004. Guía para la interpretación de cartografía. Edafología. Aguascalientes, México.

IUSS (Grupo de Trabajo WRB). 2007. Base Referencial Mundial del Recurso Suelo. Primera actualización 2007. Informes sobre Recursos Mundiales de Suelos No. 103. FAO, Roma.
Jones, J. B. Jr. and V. W. Case. 1990. Sampling, handling and analyzing plant tissue samples. pp. 389-427. In: R. L. Westerman (ed.). Soil testing and plant analysis. Soil Sci. Soc. Am. Madison, WI, USA. ISBN: 0891187936.

Kafkafi, U. 2008. Functions of the root system. pp. 13-40. In: M. Raviv and J. Heinrich Lieth (eds.). Soilless culture: Theory and practice. Elsevier. Amsterdam, Netherlands. eBook ISBN: 9780080556420.

Milagros-Garrido, E., T. García-Rujano, A. Torres, E. Sangronis, J. A. Martínez, L. C. Chaparro y L. Sánchez. 2013. Análisis de las características físicas y químicas del fruto de mango (Mangifera indica L.) "Bocado" de tres localidades del Estado Cojedes, Venezuela. Rev. Venez. Cienc. Tecnol. Aliment. 4: 189-206.

Mellado-Vázquez, A., S. Salazar-García, C. A. Treviño-de la Fuente, I. J. L. González-Durán y A. López-Jiménez. 2012. Composición y remoción nutrimental de frutos de mango 'Haden' y 'Tommy Atkins' bajo producción forzada. Rev. Mex. Cienc. Agríc. 3: 925-941.

Peralta-Antonio, N., A. E. Becerril-Román, A. Rebolledo-Martínez y D. Jaén-Contreras. 2015. Estado nutricional foliar de tres cultivares de mango fertilizados con abonos orgánicos. Idesia 33: 65-72. doi: http://dx.doi.org/10.4067/S071834292015000300010 .

Pérez-Barraza, M. H., V. Vázquez-Valdivia, J. A. Osuna-García, A. Ríos-Torres y G. López-Arriaga. 2007. Diagnóstico del cultivo del mango en Nayarit. INIFAP, CIRPAC. Campo Experimental Santiago Ixcuintla. Folleto Técnico No. 7, Santiago Ixcuintla, Nayarit, México.

Salazar-García, S., J. L. I. González-Durán y L. M. Tapia-Vargas. 2011. Influencia del clima, humedad del suelo y época de floración sobre la biomasa y composición nutrimental de frutos de aguacate "Hass" en Michoacán, México. Rev. Chapingo Serie Hortic. 17: 183-194.

Salazar-García, S., G. Santillán-Valladolid, E. F. HernándezValdés, R. Medina-Torres, M. E. Ibarra-Estrada y R. GómezAguilar. 2014. Efecto a corto plazo de la fertilización de sitio específico en mangos 'Kent' y 'Tommy Atkins' cultivados sin riego. Rev. Mex. Cienc. Agríc. 5: 645-659.

Sergent, E. 1999. El cultivo del mango (Mangifera indica L.): botánica, manejo y comercialización. Universidad Central de Venezuela. Colección monografías 72. Caracas, Venezuela. ISBN: 980001389X, 9789800013892.

SIAP (Servicio de Información Agroalimentaria y Pesquera). 2017. Secretaría de Agricultura, Ganadería, Desarrollo Rural, Pesca y Alimentación, México. http://www.siap.gob.mx (Consulted: February 09, 2019).

Stassen, P. J. C., H. G. Grove, and S. J. Davie. 2000. Uptake, distribution and requirements of macro elements in 'Sensation' mango trees. Acta Hort. 509: 365-374. doi: 10.17660/ ActaHortic.2000.509.42.

Xiuchong, Z., L. Guojian, Y. Jianwu, A. Shaoying, and Y. Lixian. 2001. Balanced fertilization on mango in southern China. Better Crops Int. 15: 16-19. 